\title{
A Quantitative Method to Evaluate Automated Constructability
}

\author{
Thomas M. Gatton \\ Department of Civil Engineering \\ The University of Texas at Austin \\ ECJ 5.2 \\ Austin, Texas 78712
}

\begin{abstract}
Before many construction activities may be automated, operations must be designed that are within the capabilities of the machinery. This can be accomplished by modification of either the construction operations or the actual design. There are several qualitative methods to evaluate automated constructability, or the amenability that construction tasks have to automation, and identify which construction tasks could be automated. Although these methods have assisted in identifying construction tasks with potential for automation, they are largely ineffective in quantifying the characteristics of a design that impact task requirements and amenability to automation. A quantitative method is needed to evaluate and measure automated constructability. This paper presents a quantitative method to evaluate the automated constructability of a design and assist in "Designing for Construction Automation". This method analyzes design characteristics through a process of reverse construction and converts them into construction requirements. The construction requirements contain motion, accuracy, stability, and environmental characteristics of the construction task. These characteristics are converted into a "task manifold chart" and a "task performance environment chart", which comprise the automated constructability criteria . Certain patterns of automated constructability criteria indicate automated constructability and technical feasibility for automation in the performance of that task. This paper describes the development of a quantitative method to evaluate automated constructability.
\end{abstract}

\section{Introduction}

As machine technologies have advanced, more effort has been made to apply them to construction tasks. The first generation of construction robots were employed in on-site construction operations in only specific applications whose manipulative requirements were within the capabilities of robots [GATTON\&BOYER88]. Although technical feasibility has been demonstrated for these applications, the cost has been relatively expensive. The use of a dedicated robot to perform a specific construction operation can be justified by the size, quantity and repetition of that operation [SKIBNIEWSKI88].

The second generation of construction automation involves modularization of robotic components and work elements [HASEGAWA85]. This requires separation of an individual robot component's manipulative characteristics. The underlying concept is to allow a set of modules that have standardized interfaces to be configured into a whole system that has the manipulative capabilities necessary to perform a given task. This modular approach has expanded capabilities, but also reveals that many operations are beyond machine capabilities and are too complicated for automation. The operations that resist automation exhibit a lack of automated constructability.

Various qualitative methods have been developed to analyze construction tasks, to evaluate their automated constructability and to select construction tasks for automation. These methods and the criteria used have been surveyed by Tucker and Petersen [TUCKER\&PETERSEN89]. Although qualitative evaluations have been helpful, they do not provide quantitative information concerning the characteristics of construction tasks and relevant design characteristics that affect automated constructability. A quantitative method is needed to provide this information and assist in design and modification for construction automation. The factors that affect the requirements for construction tasks and determine the ease with which a task can be automated must be quantified in order to identify what tasks can be automated and what tasks are obstacles to automation.

The ease with which a product can be manufactured has been called "manufacturability." In the construction industry, "constructability" is the term which refers to the ease with which construction can be accomplished using traditional methods and techniques. "Automated constructability" refers to the amenability of construction tasks to automation. Automation, in the context of this research, includes the use of a single general purpose manipulator, mobile device, or combination thereof that is controlled either automatically or manually to perform a task. The use of automated equipment in construction has been largely limited to 
teleoperated devices such as cranes, earthmoving equipment, automated hand tools, and other types of material handling and processing devices.

This research addresses the issue of automated constructability. A quantitative method to evaluate automated constructability of construction tasks is presented. This method analyzes design characteristics through a process of reverse construction and converts them into construction requirements. The construction requirements contain motion, accuracy, stability, and environmental characteristics of the construction task. These characteristics are converted into a "task manifold chart" and a "task performance environment chart", which comprise the automated constructability criteria . Certain patterns of automated constructability criteria indicate automated constructability and technical feasibility for automation in the performance of that task.

\subsection{Scope and Limitations}

The method developed is limited to evaluation of construction tasks for performance by the following types of automated equipment:

1. General purpose manipulators (robots) controlled by manual or automatic devices

2. Mobile, general purpose manipulators (robots) controlled by manual or automatic devices.

3. Mobile mechanisms controlled by manual or automatic devices.

4. Single, general purpose manipulators or mobile mechanisms performing a single task.

These limitations include a wide variety and various levels of automation. Many traditional machines as well as new robotic technologies are included in these categories. As new machine capabilities develop, methods will need to be developed which analyze tasks performed by multiple cooperating machines.

The method is intended to evaluate the technical feasibility of performing a construction task with a single piece of automated equipment. It does not address economic, safety, productivity, and other similar issues.

\section{A Quantitative Method To Evaluate Automated Constructability}

The construction of a specific design is accomplished through performance of construction operations related to characteristics of the design. These construction operations involve manipulation of tools and materials to place, shape, and connect materials and components as specified in the design. The manipulation of tools and materials consists of individual construction tasks which have motion and accuracy requirements. During performance of construction tasks, the existing environmental characteristics, within which the machines must perform the construction tasks, and stability characteristics must be evaluated. These requirements and characteristics comprise the automated constructability criteria. By evaluating the automated constructability criteria, the automated constructability, or amenability of the task to automation, can be determined.

The automated constructability criteria are identified through automated constructability issues. Automated constructability issues are considerations about construction task performance that identify obstacles to automated construction. Design characteristics are mapped into the automated constructability criteria through automated constructability issues. Automated constructability criteria consist of the motion and accuracy requirements to perform the task and the stability and environmental characteristics that occur during and subsequent to performance of the task. The automated constructability criteria are generated through a process to be introduced of reverse construction of the design.

Evaluation of a task's automated constructability criteria determines the automated constructability of a single construction task. Various sequences of construction tasks can be selected and evaluated to determine a sequence in which all tasks exhibit automated constructability. Where no sequence of tasks has automated constructability, the individual tasks lacking automated constructability can be identified and evaluated to assist in determining design changes that provide automated constructability. It is important to note that the method will only indicate if a task has automated constructability and the design characteristics that generate task requirements, stability factors and environment that lack automated constructability. The designer must determine which changes will provide automated constructability.

This section introduces a quantitative method to evaluate the automated constructability of construction tasks that comprise construction operations. Section 2.1 introduces the design characteristics affecting automated constructability. Section 2.2 identifies automated constructability issues. Section 2.3 identifies the criteria identified in this work which determine automated constructability. These criteria are identified through automated constructability issues and include task motion and accuracy requirements as well as stability and environment characteristics encountered during and subsequent to task performance. Section 2.4 introduces the reverse construction method to generate the automated constructability criteria from the design characteristics. Finally, Section 2.5 explains use of the method to generate automated constructibility evaluations. 


\subsection{Design Characteristics Affecting Automated Constructibility}

The design characteristics affecting automated constructability exist at two different levels. The first level is that of the individual component. The second level of characteristics affecting automated constructability is that of relationships between individual components (multicomponent level).

The individual component characteristics identified in this research that affect automated constructability are:

1. Rigidity/Viscosity,

2. Elasticity,

3. Strength,

4. Weight,

5. Geometry or shape, and

6. Tolerances in dimensions.

The characteristics identified in this research affecting automated constructability at the multicomponent level, or relationships between individual components, are:

1. Geometric relationships,

2. Frictional forces,

3. Fasteners, and

4. Connections.

\subsection{Automated Constructibility Issues}

Automated constructibilitiy issues are concerns that must be addressed during performance of a construction task to identify any obstacles to automation of the construction task. These issues include:

1. Contacted component stability,

2. Manipulated component/tool stability,

3. Construction Sequencing,

4. Local component movement, or orientation and movement,

5. Global component movement, or material feeding,

6. Equipment placement, stability, mobility, and operation,

7. Deflection,

8. Self-support during grasping, manipulation and placement, and

9. End effector grasping requirements.

These characteristics are used in Section 3.4 to map the automated constructibility criteria into the design characteristics

\subsection{Automated Constructibility Criteria Considered in this Work}

Automated constructability criteria consist of the construction task motion and accuracy requirements and the stability and environment characteristics occuring during the performance of the construction task. The construction task motion requirements and the Task Motion Chart are presented in Section 3.3.1. The accuracy requirements and Task Accuracy Chart are presented in Section 3.3.2 The stability characteristics which affect automated constructability are identified in Section 3.3.3. The task motion, accuracy and stability and requirements are combined into the Task Manifold Chart presented in Section 3.3.4. The environmental characteristics encountered during task performance and the Task Performance Chart are presented in Section 2.3.6.

\subsubsection{Construction Task Motion Chart}

The performance of an automated construction task may require translation and orientation of an object in space. This motion must be performed within a certain accuracy to guarantee accomplishment of the goals of the task. Motion and accuracy requirements can be described for a local task through homogenous coordinate transformation matrices [FOLEY82\&PAUL83]. Generation of motion and accuracy requirements from configuration space [LATOMBE89] is described in other research. For simplification, the models shown here will be in two dimensional space of the $\mathrm{X}$ and $\mathrm{Z}$ planes with one rotational degree of freedom around the $\mathrm{Y}$ axis. Further simplification will be through the approximation of curves with straight line segments with rotation proportional to the distance traveled along that line. Issues of velocity and acceleration requirements will not be addressed as manipulators are capable of minimally matching humans in this area. Rigorous mathematical modeling is left for researchers in the field of analytic geometry. Figure 3 demonstrates the task motion chart to describe the motion necessary to move the triangle from its position in Figure 1 to the position shown in Figure 2. 


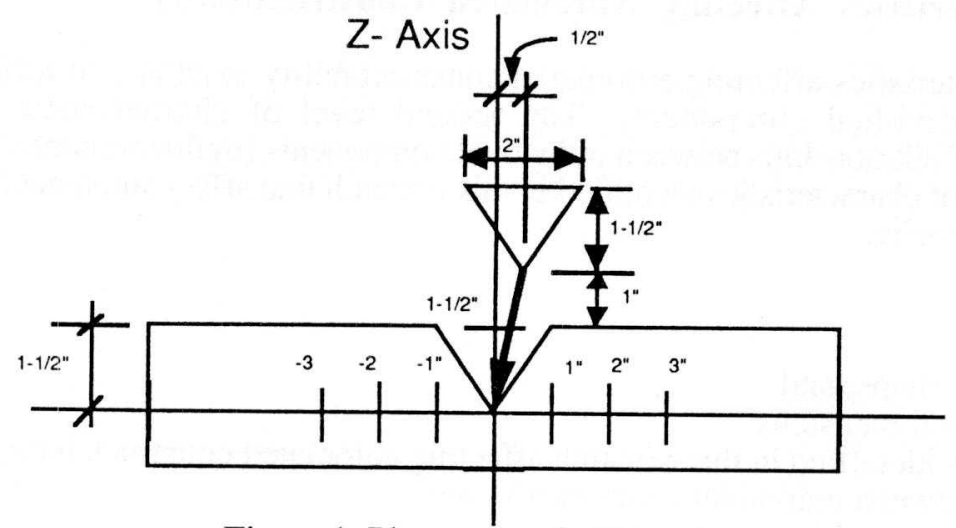

Figure 1 Placement of a Triangle

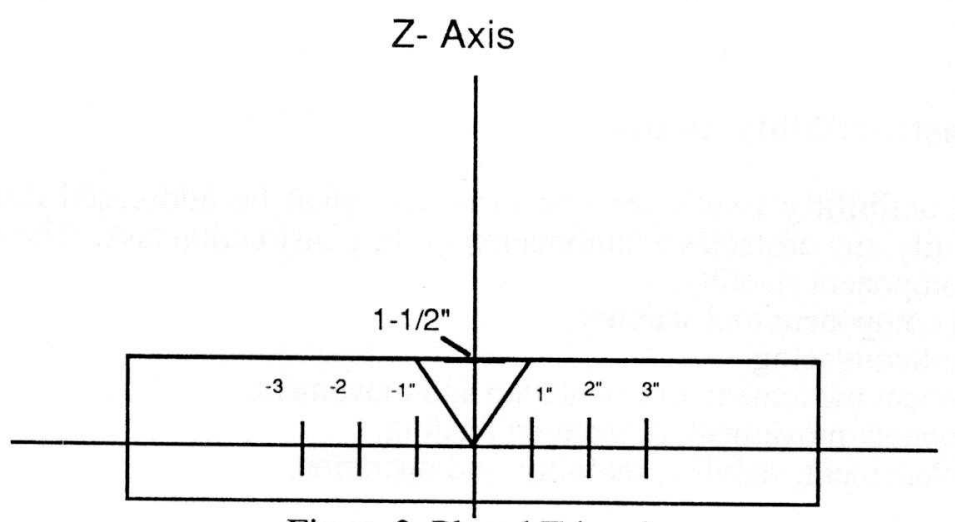

Figure 2 Placed Triangle

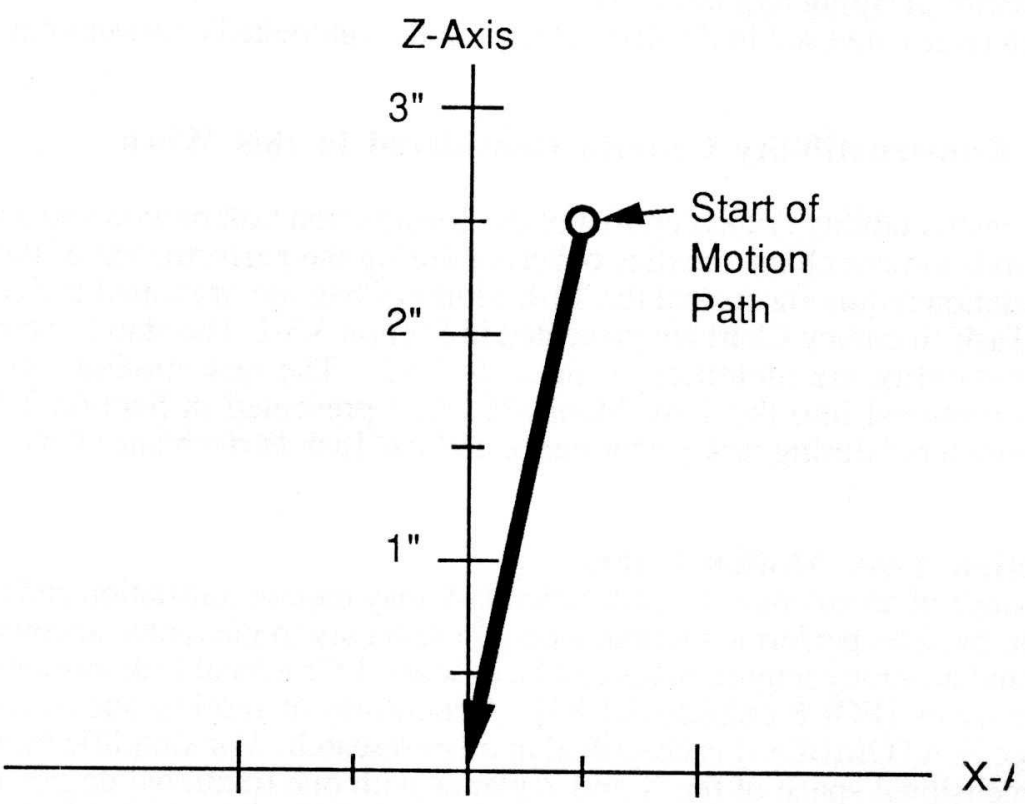

Figure 3 Task Motion Chart for Triangle Placement

The essential data to describe the straight path of motion include:

1. The start position of the path,

2. The finish position of the path,

3. The amount of rotation occuring over the path. 


\subsubsection{Task Accuracy Chart}

When a motion is performed, there is often an error between the desired position and the actual position of the manipulator. During the performance of a task, there is also error in the known location of the manipulated and contacted components. The accumulation of these errors and their affect on task performance must be checked to insure that a task can be successfully completed. A modeling method is needed to convey the accuracy requirements for each task necessary to construct a specific design. This is accomplished through the task accuracy chart which incorporates tolerances in the manipulated and contacted component in determining the local free space within which the manipulator must perform the task. This model can be developed by surrounding the components to be assembled with a tolerance layer as shown in Figure 4 . The placed component is then translated around the mating component to define the C-obstacle and the outline of the accuracy manifold. To illustrate the accuracy manifold and develop the task accuracy chart, reconsider the task of moving the 2 " by $1-1 / 2^{\prime \prime}$ triangle, shown in Figure 1, from its original position and through a straight path to its final position $1 / 2$ " in the negative $\mathrm{X}$ direction and $2-1 / 2$ " in the negative $\mathrm{Z}$ direction as shown in Figure 2. The motion path to accomplish this is shown in Figure 3. The shaded region in Figure 4 shows a tolerance error of plus or minus 1/8" for the drawings shown previously in Figures 1 and 2 . This results in a total shaded region, or tolerance error, of $1 / 4 "$. In the lower drawing of Figure 5 , the triangle is placed where the tolerance regions make contact. From Figure 4, a C-obstacle is generated to form the task accuracy chart shown in Figure 5. The C-obstacle generated to form the task accuracy chart is translated from its origin the amount which the tolerance triangle was dislocated from its final position in Figure 1. This example demonstrates the concepts of the accuracy manifold and the task accuracy chart. The accuracy manifold describes the local envelope of space surrounding the task that the manipulator must stay within while performing the task. It is the free space remaining when the tolerance of the components is integrated into the generation of the $\mathrm{C}$-obstacle. The task can be described as a single point moving through the accuracy manifold. The task accuracy chart will be combined with the task motion chart, previously presented in Section 3.3.2.1 and the stability characteristics chart, to develop the task manifold chart in Section 3.4
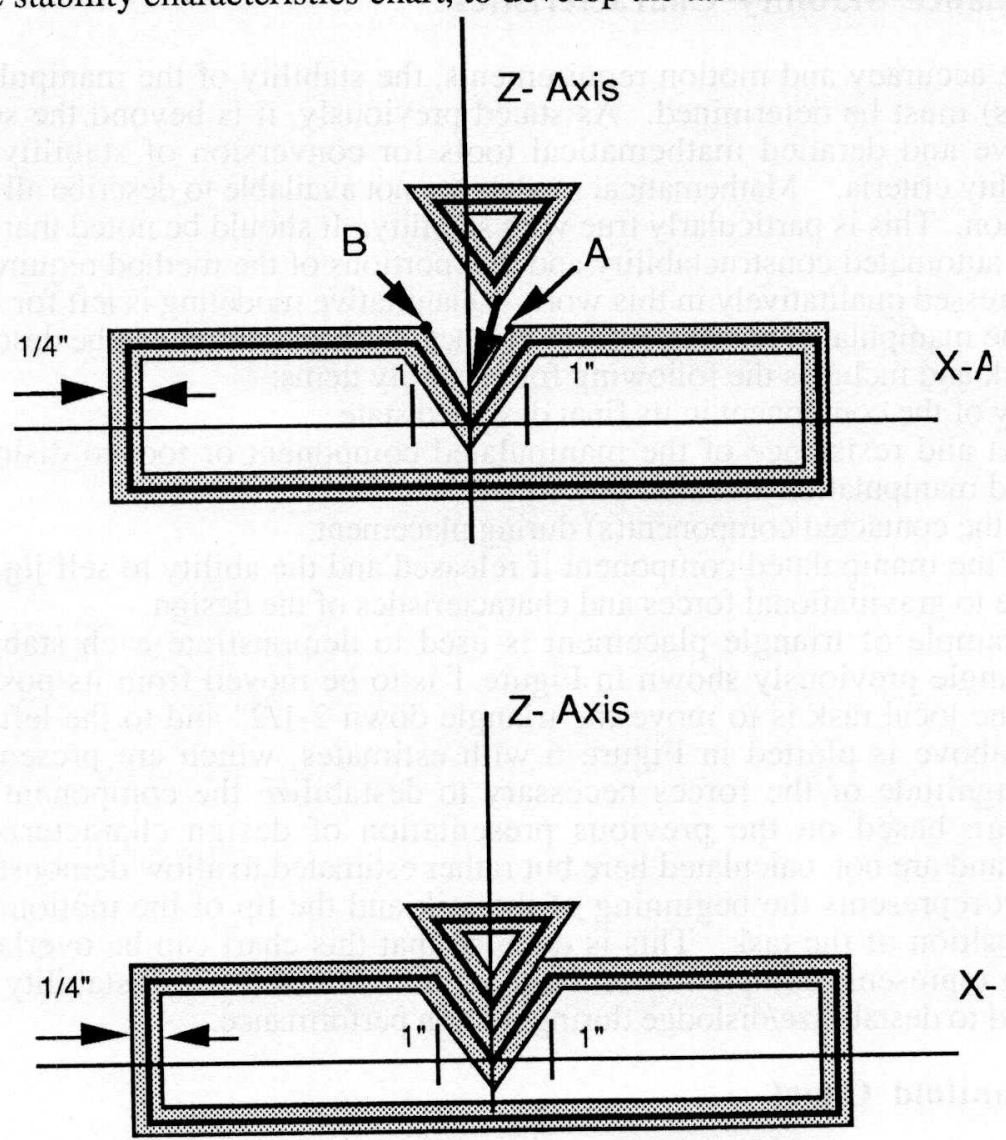

Figure 4 Triangle and Component Tolerance 


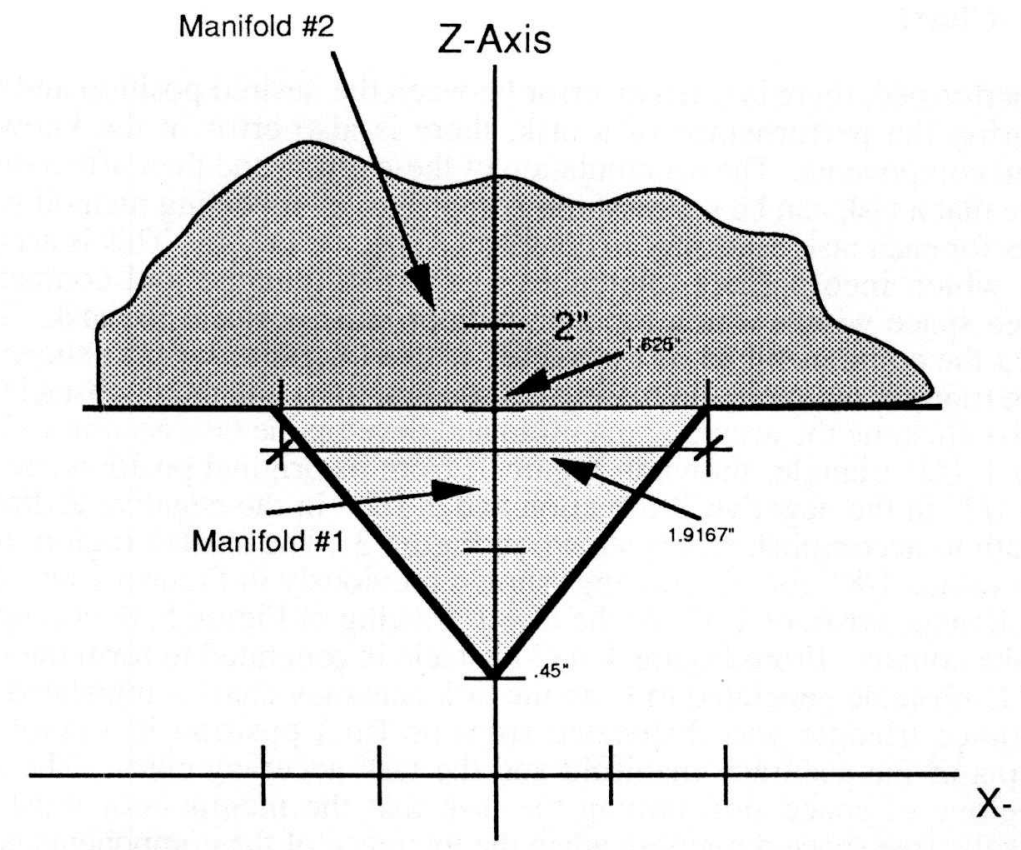

Figure 5 Task Accuracy Chart for Triangle Placement

\subsubsection{Task Performance Stability Characteristics}

In addition to the accuracy and motion requirements, the stability of the manipulated component and contacted component(s) must be determined. As stated previously, it is beyond the scope of this work to present comprehensive and detailed mathematical tools for conversion of stability characteristics into automated constructability criteria. Mathematical models are not available to describe all of the operations that occur during construction. This is particularly true with stability. It should be noted that this work focuses on a method to determine automated constructability and that portions of the method require additional research. Stability issues are addressed qualitatively in this work. Quantitative modeling is left for future research.

The stability of the manipulated component and contacted component(s) can be described over the time it takes to perform the task and includes the following four stability items:

1. The stability of the component in its final designed state

2. Self-support and resistance of the manipulated component or tool to dislodging forces during grasping and manipulation and after placement.

3. Stability of the contacted component(s) during placement.

4. Stability of the manipulated component if released and the ability to self jig, or reach the desired position due to gravitiational forces and characteristics of the design.

The previous example of triangle placement is used to demonstrate each stability item. Figure 3 illustrates how the triangle previously shown in Figure 1 is to be moved from its position to the position shown in Figure 2. The local task is to move the triangle down 2-1/2" and to the left $1 / 2$ ". Each of the 4 stability items listed above is plotted in Figure 6 with estimates, which are presented in the next four paragraphs, of the magnitude of the forces necessary to destabilize the component or material. These approximate values are based on the previous presentation of design characteristics and automated constructability issues and are not calculated here but rather estimated to allow demonstration of the method. The origin of the chart represents the beginning of the task and the tip of the motion arrow represents the completion or final position of the task. This is done so that this chart can be overlayed on the accuracy manifold whose origin represents completion of the motion. Each of the four stability items are plotted for estimated force required to destabilize/dislodge during motion performance.

\subsubsection{The Task Manifold Chart}

The task manifold chart combines the task motion chart, the task accuracy chart, and the task stability chart into a single chart. This chart is then used in conjunction with the task performance environment chart, 


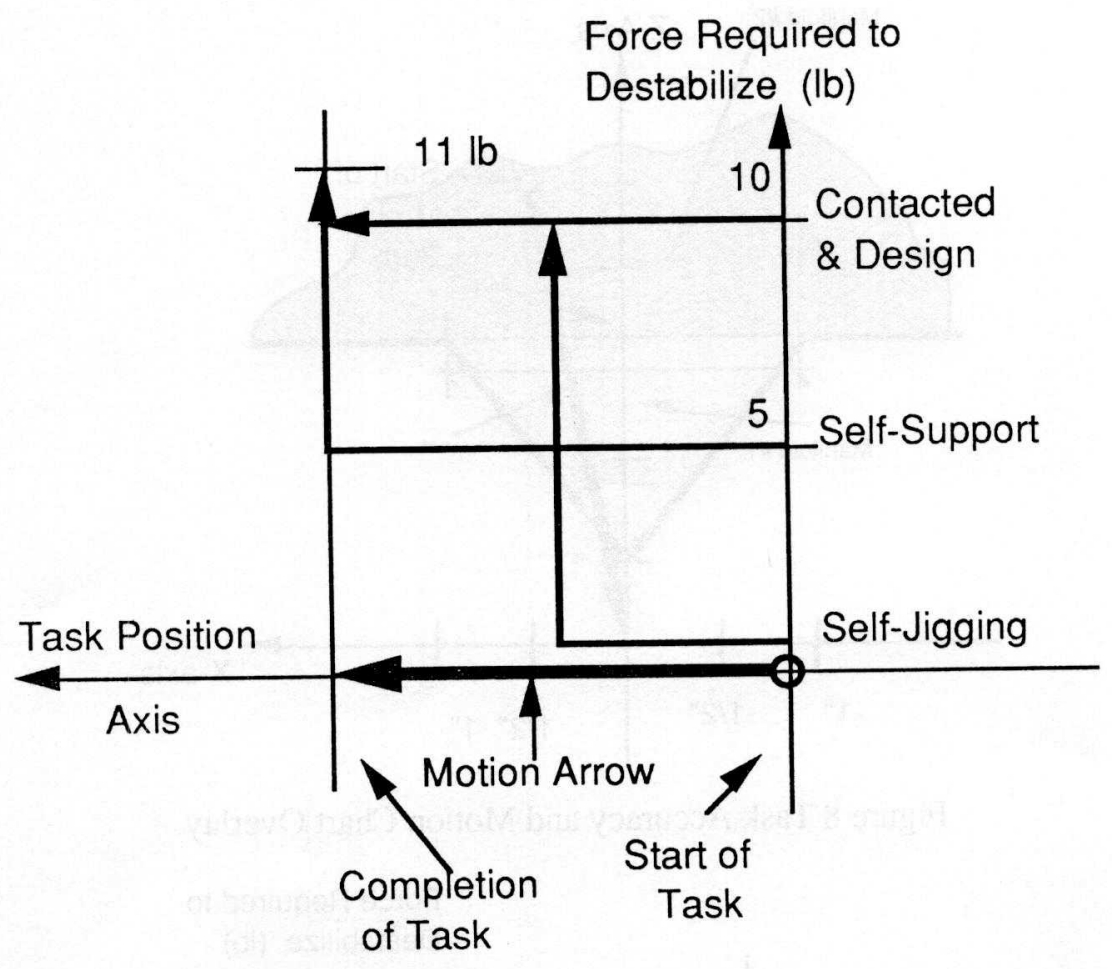

Figure 6 Task Stability Chart for Triangle Placement

presented later in Section 3.5 to evaluate the automated constructability of a task. The task manifold chart is demonstrated with the triangle placement example in Figure 9. This example demonstrates the task motion, accuracy, and stability charts. This example of the triangle uses the task motion chart of Figure 3 , the task accuracy chart of Figure 5, and the task stability chart of Figure 6. These charts are combined to demonstrate the task manifold chart.

Figure 9 is the task manifold chart for the previous example of placing a triangle as shown in Figure $1 \& 2$. The first step in generating the task manifold chart is to overlay the task motion chart with the task accuracy chart. This is shown in Figure 8 . The intersection of the motion path and the edge of the accuracy manifold indicate where there is possible contact between the manipulated and contacted component. Next, the combined motion and accuracy chart is rotated to align the motion path/arrow with the Task Position axis of the stability chart. This combination results in the task manifold chart, as shown in Figure 9. The stability characteristics during task performance can then be evaluated along the motion path and accuracy manifold to determine the task's automated constructability for this part of the automated constructability criteria.

The task manifold chart and the task performance environment characteristics comprise the automated constructability criteria. The task performance environment characteristics are presented in the next section. A method to generate the automated constructability criteria from the design characteristics through reverse construction is presented in Section 3.6. The rules to evaluate the automated constructability criteria for automated constructability are presented in Section 3.7.

\subsubsection{The Task Performance Environment Characteristics}

The environment must be evaluated to determine any characteristics which are not amenable to equipment performing a task. These issues include:

1. Can equipment be placed in a location from which the task can be performed?

2. Is sufficient stability provided to the equipment for performance of the task?

3. From a global perspective, can the equipment be moved into position?

4. From a global perspective, can materials be fed to the equipment and moved to the final task position?

5. Are the forces of the task beyond machine capabilities?

These issues are represented by the Task Performance Environment Chart show in Figure 9. 


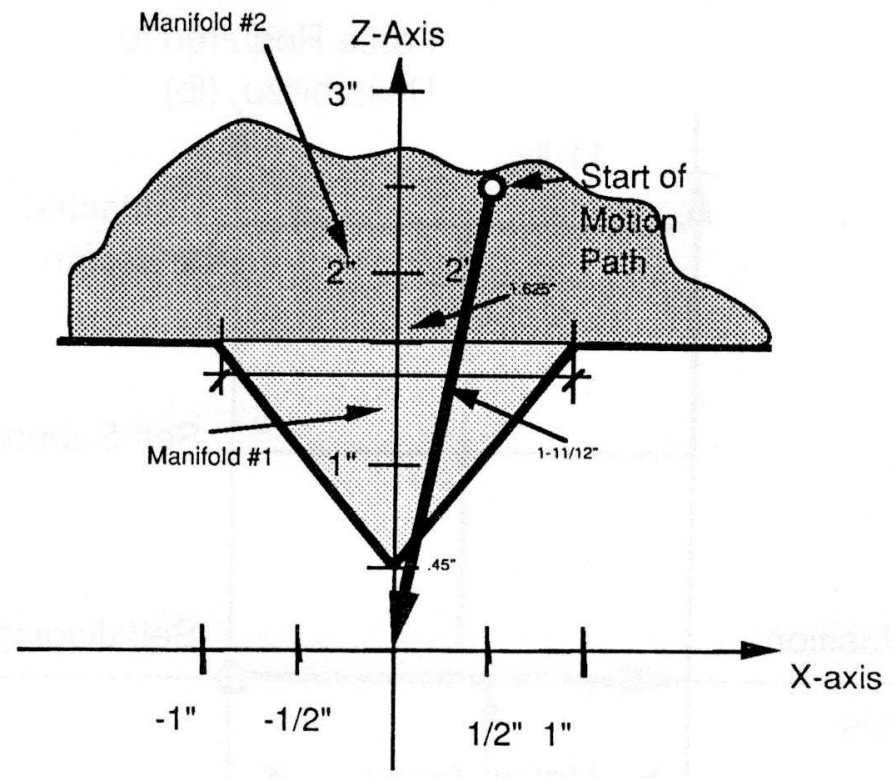

Figure 8 Task Accuracy and Motion Chart Overlay

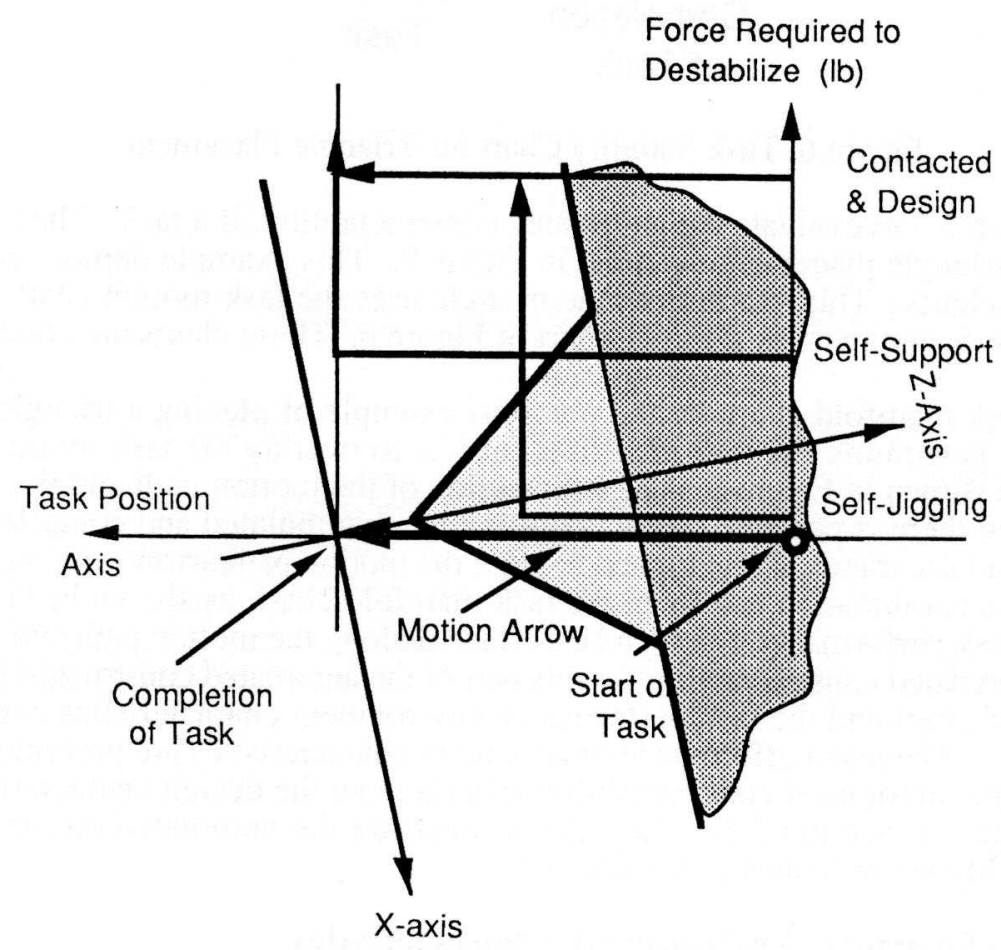

Figure 9 Task Manifold Chart for Triangle Placement

\subsection{Reverse Construction Method to Generate Automated Constructibility Criteria}

In this section, the automated constructibility criteria are generated from the relevant design characteristics through a method which will be introduced as "reverse construction". The method generates a reverse motion path for the local task. This path is reversed to generate the task motion requirement for the automated constructibility criteria. Other design characteristics are analyzed during and subsequent to task performance to generate the remainder of the automated constructibility criteria.

A technique to generate assembly plans has been developed at Carnegie Mellon University. This technique 


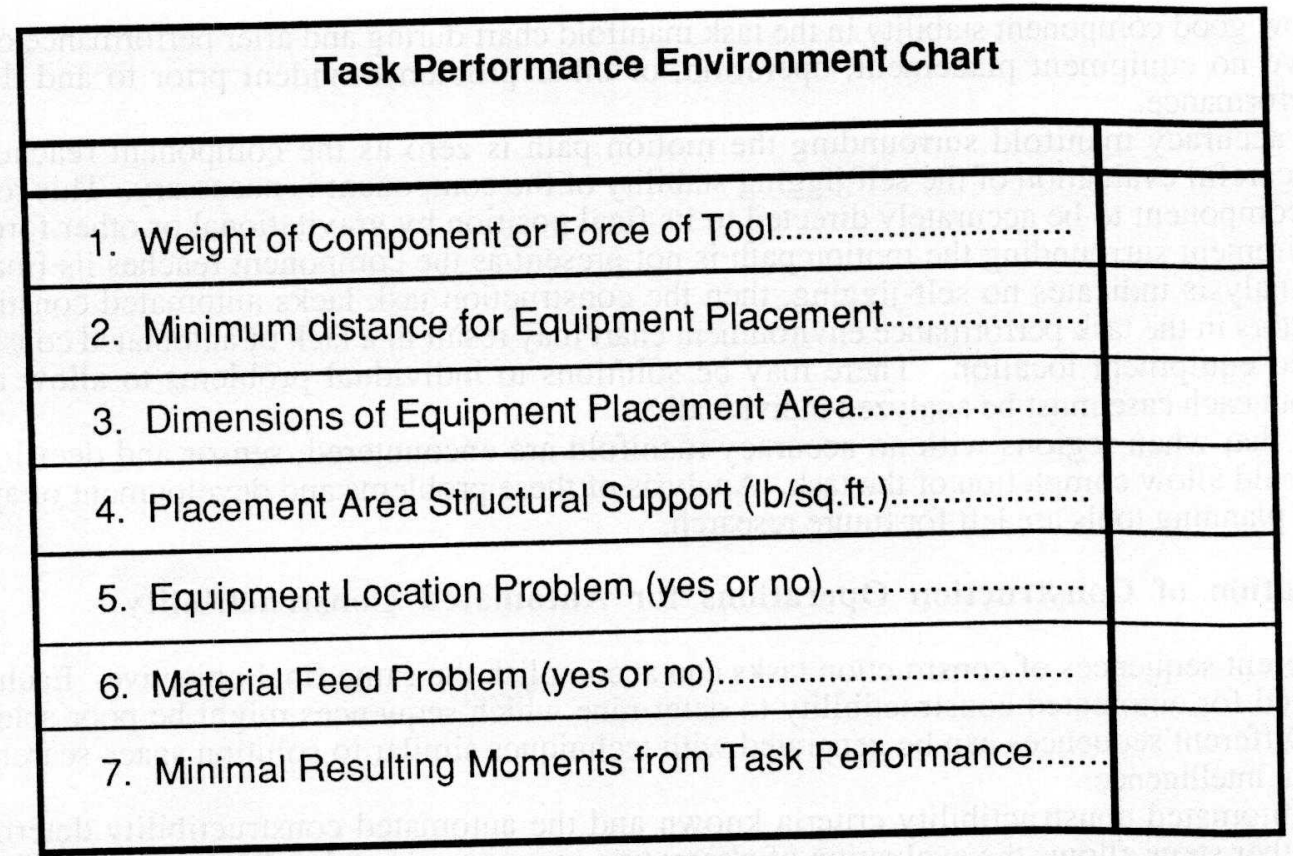

Figure 9 Task Performance Environment Chart

transforms the problem of generating assembly sequences "...into the problem of generating disassembly sequences in which the disassembly tasks are the inverse of feasible assembly tasks." [HOMEN88]. During this disassembly, checks are performed to determine the mechanical and geometric feasibility and stability of the operation.

The method presented here to evaluate design characteristics for "automated constructibility" consists of reverse constructing the design to generate the motion path and analyzing relevant design characteristics during and subsequent to the performance of the task. This method generates the automated constructibility criteria which can be evaluated for automated constructibility of the task.

The method for reverse construction and generation of automated constructibility from design characteristics consists of the following steps:

1. Check the C-obstacles of the design to identify a component for reverse construction.

2. If a component comprised of rigid material is being placed, generate a motion path of minimal tolerance interference for reverse construction. The angle at which the motion path most quickly reaches the accuracy manifold is generally the best path to eliminate tolerance interference.

3. If a component comprised of rigid material is not being placed, generate the tool motion path of minimal tolerance interference from tool manipulation requirements.

4. Generate the accuracy requirements for the path of motion from design characteristics of geometric relationships and tolerances.

5. Generate the stability information from frictional, connector, strength, geometry, viscosity, and weight characteristics of the component or tool that is being manipulated.

6. Generate the task performance environment chart information from geometric relationships between components and structural relationships between components.

\subsection{Use of Method to Evaluate Automated Constructibility}

The characteristics of the task manifold chart and the task performance environment chart can be examined to evaluate the automated constructibility of each construction task. The task manifold chart contains information concerning the motion path that the manipulator must perform and the accuracy manifold of the motion path required to complete each construction task. It also contains stability characteristics that must be evaluated. The task performance environment chart helps to determine if equipment can be located to perform a construction task, the support and size limitations of a location, geometric constraints on material feeding and equipment operation, and the loads introduced during performance of the construction task.

Construction tasks that have good automated constructibility are those which:

1. exhibit a large accuracy region completely surrounding the path of motion, 
2. show good component stability in the task manifold chart during and after performance of the task,

3. have no equipment placement, operation, or other problems evident prior to and during task performance.

When the accuracy manifold surrounding the motion path is zero as the component reaches its final position, then careful evaluation of the self jigging stability of the component is necessary. This refers to the ability of the component to be accurately directed to its final position by gravitational or other forces. If the accuracy requirement surrounding the motion path is not present as the component reaches its final position and stability analysis indicates no self-jigging, then the construction task lacks automated constructibility. Further, indicators in the task performance environment chart may result in a lack of automated constructibility for the planned equipment location. There may be solutions to individual problems to allow automated construction, but each case must be analyzed individually.

It is likely that when regions with no accuracy manifold are encountered, sensor and decision making capabilities would allow completion of the task. Analysis of these problems and development of appropriate diagnostic and planning tools are left for future research.

\subsubsection{Evaluation of Construction Operations for Automated Constructibility}

Many different sequences of construction tasks can accomplish the same final objective. Each sequence can be evaluated for automated constructibility to determine which sequences might be poor selections for automation. Different sequences can be generated with techniques similar to solution space searching in the field of artificial intelligence.

With the automated constructibility criteria known and the automated constructibility determined, the addition of further steps allows the evaluation of alternative sequences of tasks. These steps, added to those presented in Section 3.7, consist of:

7. Evaluate the task manifold chart and the task performance environment chart to determine the automated constructibility of the construction task,

8. Determine if this is the last component at this level of task sequence identified by C-obstacle analysis that can be reverse constructed. If it is the last component and the task is not amenable to automated construction, then redesign must take place to provide automated constructibility of the task. Exit the reverse construction method at this point.

9. Determine if this is the last component of the design and if the construction task is amenable to construction automation. If so, then this design and this sequence of construction tasks is amenable to construction automation. Exit the reverse construction method at this point.

10. If this is not the last construction task and it is amenable to automated construction, loop back to step 1 of the method presented in Section 3.7 and identify the next component for reverse construction

11. If this is not the last construction task and it is not amenable to automated construction, return the component to its original place in the design and loop back to step 1 and identify the next component for reverse construction.

These steps allow the generation of various sequences of construction tasks and evaluation of their automated constructibility.

\section{REFERENCES}

Foley, J., \& Van Dam, A., "Fundamentals of Interactive Computer Graphics,"Addison-Wesley Publishing Company, Reading, Massachussetts, 1982.

Gatton, T., \& Boyer, L. "Design for Automated Construction," Proceedings of the Fifth International Symposium on Robotics in Construction, Tokyo, Japan, June, 1988.

Hasegawa, Y., "Industrial Robot Standardization," in "Handbook of Industrial Robotics," John Wiley and Sons, New York, New York, 1985.

Homen de Mello, L., Sanderson, A., "Automatic Generation of Mechanical Assembly Sequences," CMU-RITR-88-19, Carnegie Mellon University, Pittsburgh, PA, 1988.

Latombe, J., "Global Path Planning Approaches for Material Movements in a Worksite," CIFE Technical Report No. 0003, Stanford University, Palo Alto, California, 1989.

Paul, R., "Robot Manipulators: Mathematics, Programming, and Control," The MIT Press, Cambridge, Massachusetts, 1983.

Skibniewski, M., "Robotics in Civil Engineering," Computational Mechanics Publications, Boston, Massachusetts, 1988.

Tucker, R., \& Petersen, C., "A Methodology for Identifying Automation Opportunities in Industrial Construction," A Report to the Construction Industry Institute, The University of Texas at Austin, Austin, Texas, November, 1989. 